\title{
A Nonlinear Control Algorithm for Improving Settling Time in Systems With Friction
}

\author{
Brian A. Bucci, Daniel G. Cole Member, IEEE, Stephen J. \\ Ludwick Member, IEEE, and \\ Jeffrey S. Vipperman
}

\begin{abstract}
A nonlinear control algorithm is proposed that greatly reduces settling time in precision instruments with rolling element bearings. Reductions of $80.5-87.4 \%$ in settling time were achieved when settling to within $3-100 \mathrm{~nm}$ of the commanded position. Final settling of such systems is typically impacted by the nonlinearity in the pre-rolling friction regime, which manifests as a hysteretic stiffness. Consequently, the integral term in the controller can take a long time to respond. In this work, the Nonlinear Integral Action Settling Algorithm (NIASA) is presented. The nonlinear integral gain takes the form of a Dahl friction model. Since the integral gain mimics hysteretic stiffness, the output of the integral control term is instantaneously set to a large value after each direction change, greatly improving settling response. A nearly first order error dynamic results, which has a user-definable time constant. Before the algorithm can be implemented, the Coulomb friction and initial contact stiffness in the Dahl model must be experimentally determined for the stage. A sensitivity study was performed on the initial contact stiffness, which was found in other works to dictate stability of the algorithm [1, 2].
\end{abstract}

\section{INTRODUCTION}

Rapid point-to-point motion of a servo mechanism has obvious industrial applications. In these systems, the objective is to move as quickly as possible from one location to the next with no concern for the particular motion profile used. Many such mechanisms contain rolling element bearings, given their low cost and generally good performance. As industrial processes

Manuscript received October 13, 2011; revised: March 15, 2012. This work was supported by Aerotech, Inc.

B.A. Bucci, J.S. Vipperman, and D.G. Cole are with University of Pittsburgh

S.Ludwick is with Aerotech, Inc. and University of Pittsburgh 


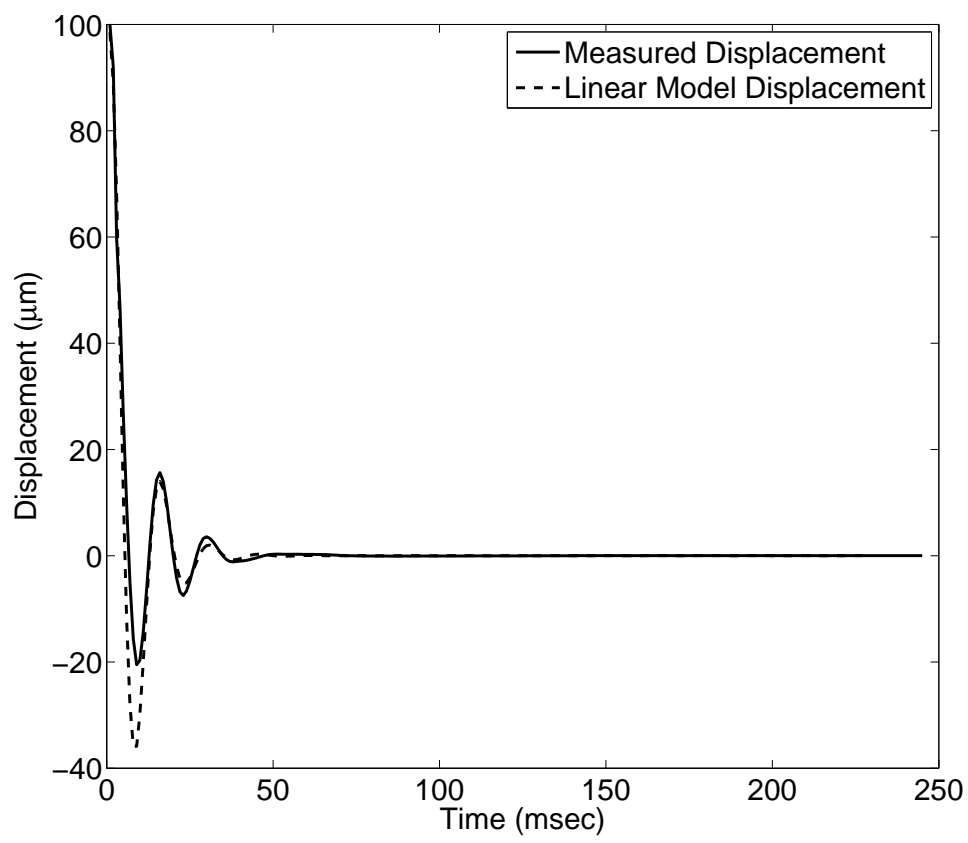

Fig. 1. Comparison of an actual servo step response error with the prediction by a linear model for a 100 micrometer step.

begin to require nanometer tolerances, the settling time of a servo after a step motion has significant impact on process throughput. For the large-amplitude servo motion, linear systems theory works quite well to predict and achieve performance for servos with rolling contacts. However, when nanometer precision is desired, the nonlinearity of pre-rolling friction tends to dominate the system response and the conventional linear methods no longer provide an accurate approximation of the system. An example of this phenomenon is illustrated through figures 1 and 2. In figure 1 a basic linear model appears to capture the majority of a servo's response to a $100 \mu \mathrm{m}$ step input with a reasonable degree of accuracy. However, if figure 1 is scaled to show only the final micrometer of settling, as shown in figure 2, it is clear that the linear model does not describe the servo behavior throughout the entire settling process. Thus, nonlinear friction must be considered if an accurate description of the entire servo settling process is to be constructed.

Another accepted method of friction compensation is the friction observer[3, 4]. The friction observer uses measured states of the system and passes them through a model of the friction process to estimate the true friction process. This estimate is used to construct a signal to exactly 


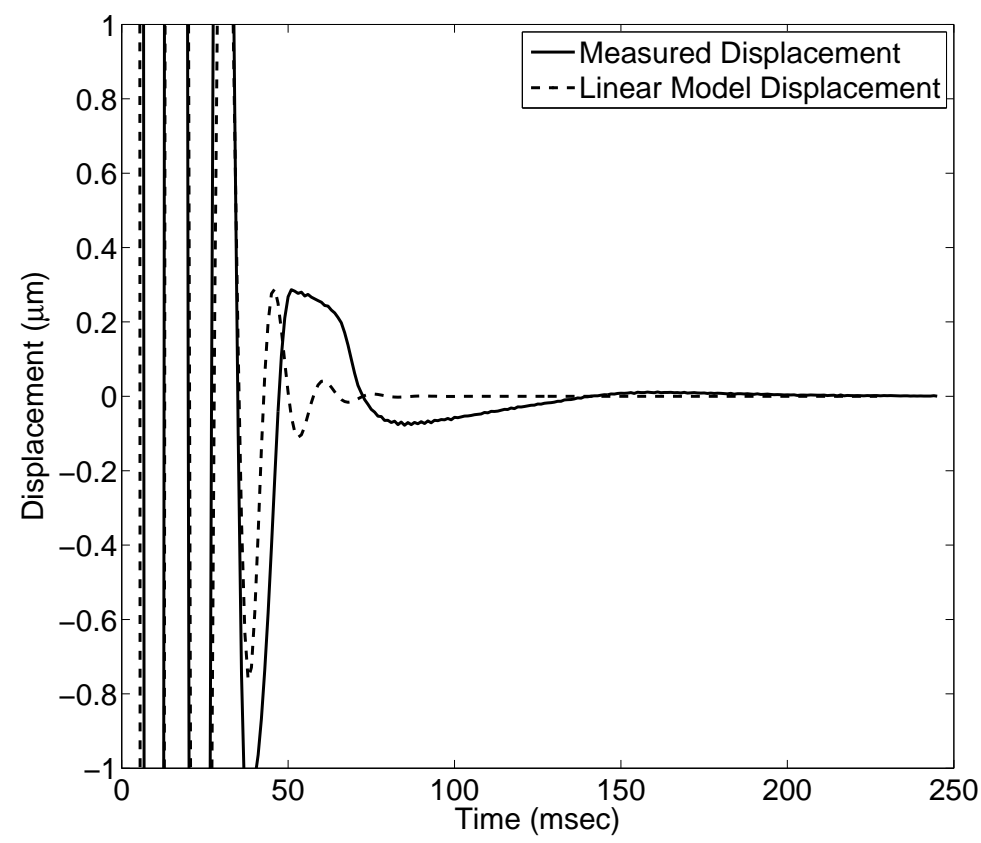

Fig. 2. Comparison of an actual servo step response with the prediction by a linear model re-scaled to focus on the final micrometer of settling.

cancel the frictional process, leaving only the inertial dynamics of the servo. Several studies have shown the ability to improve servo tracking performance with use of friction observers $[3,5,6,7,8,9,10,11,12]$. However, some of these same experimental studies have shown the friction observer to offer little or no increase in point-to-point servo performance [5, 6]. It has even been stated that it is possible that the friction observer can actually degrade point-to-point performance in some cases[6].

For the point-to-point motion problem, the main issue with the friction observer is that most analysis is based on the assumption that the true friction process can be perfectly captured by the friction model. However, constructing a perfect model of the friction process that globally describes a real servo mechanism is an impractical task. Differences between the modeled friction process and the true friction process can lead to undesirable system characteristics, such as, system instability and extended servo settling times[6]. Adaptive extensions to friction observers have been developed $[6,13,14]$. However, these efforts are not particularly concerned with the rate at which the model of the frictional process converges to the true process and 
it appears that the methods take at least several seconds to converge (much longer than the settling process). Additionally, these efforts tend to focus on tracking sinusoids which, could be considered repetitive in nature. From the authors' experience, repetitive servo motion, on a time scale of seconds to minutes, tends to cause the frictional characteristics of the servo to settle into a somewhat predictable pattern. Thus, it is not surprising that an adaptive method would be likely to perform well for these problems.

This work will present a nonlinear control method that is based on a Dahl friction model. It will begin with an overview of pre-rolling friction and modeling in section II, followed by an analysis of the settling process in Section III. Next, the Nonlinear Integral Action Settling Algorithm (NIASA) will be presented in Section IV. Finally, simulation (Section V) and experimental results (Section VI) are presented, followed by the Conclusions in Section VII.

\section{Pre-Rolling Friction}

The classical Coulomb friction model approximates friction as

$$
F_{r}=\mu F_{N} \operatorname{sgn} \dot{x},
$$

where $F_{r}$ is the force of friction, $\mu$ is the coefficient of kinetic friction, $F_{N}$ is the normal force at the contact, and $\dot{x}$ represents the relative velocity of the surfaces at the contact. When a velocity reversal occurs, this model predicts that the force of friction will instantly change direction while preserving the same magnitude. While this description is sufficient for large scale approximations, on the sub-micrometer length scale, the force of friction has been observed to have a gradual transition between opposing levels of Coulomb friction at velocity reversal[15]. The friction dynamics that occur at this time are known as pre-rolling behavior. More advanced friction models are required in this case.

The Dahl model is the simplest of the more advanced friction models, which are capable of capturing pre-rolling behavior[15]. It is expressed in differential form as:

$$
\frac{d F_{r}}{d x}=\sigma\left(1-\frac{F_{r}}{F_{C}} \operatorname{sgn} \dot{x}\right)^{i},
$$

where $\sigma$ is the initial contact stiffness, $F_{C}$ is the apparent level of Coulomb friction, and $i$ is a shape factor, typically set to $i=1$. Figure 3 shows the response of this model to an example displacement signal. As seen in figure 3, the response of the Dahl model does capture the general 


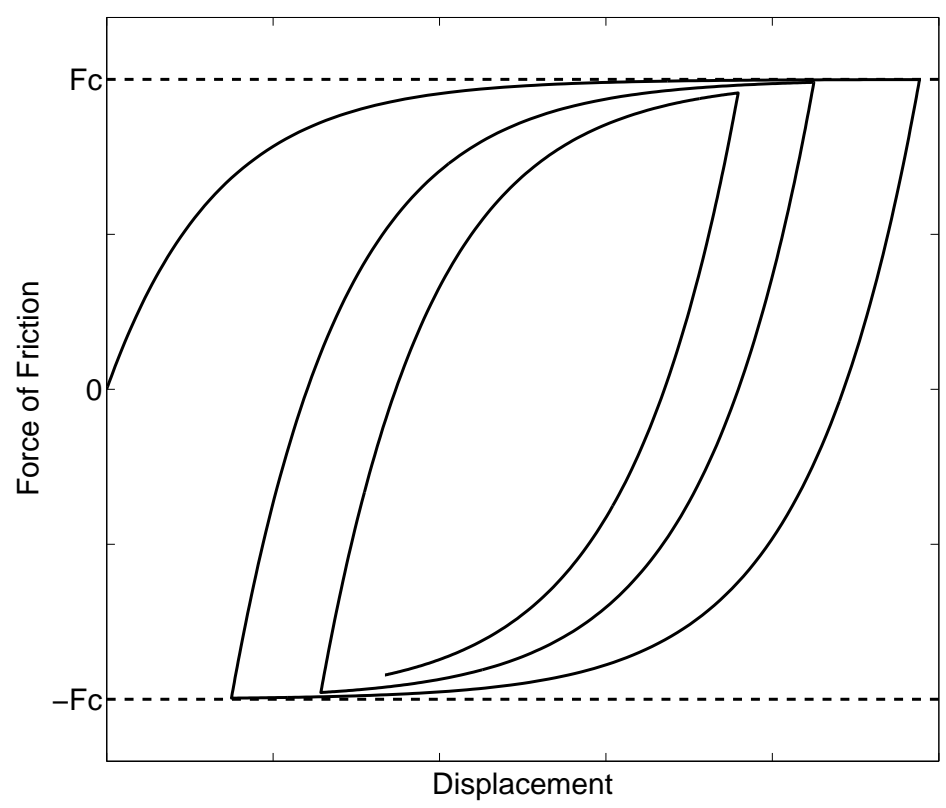

Fig. 3. Example of the type of response seen from Dahl's friction model in terms of force versus displacement.

hysteretic stiffness. Given that the proposed method of control does not require a high fidelity hysteresis model, it will be used to-describe the force of friction in this work.

\section{Analysis of the Settling Process}

Before designing a control algorithm to address the servo settling problem, it is necessary to study the forces acting on the system during settling. The simple equation of motion for a controlled servo subject to friction is

$$
m \ddot{x}+F_{r}=u(t),
$$

where $m$ is the mass or inertial component, $F_{r}$ is the friction process, and $u(t)$ is the control signal. This simplified equation assumes that the electrical dynamics of the servo and the contribution of the viscous damping are negligible. For this work, all of these assumptions are true and the only significant system dynamics are inertial and frictional. Since the input, $u(t)$, and the moving mass of the system, $m$, are known, and the acceleration, $\ddot{x}$ can be measured, it is possible to separate the inertial and frictional components of the system response. 
For example, this analysis is performed on data gathered from an ALS-130-H linear stage made by Aerotech Inc. Figure 4 shows a typical "move-and-settle" motion profile for a displacement change of $5 \mathrm{~mm}$. This work is concerned with the settling portion, after the displacement command ends. In figure 5 the input force, the response from the inertial component, and the response from the frictional component are all presented versus time. In figure 5, the vertical line at $154 \mathrm{~ms}$ indicates the time when the commanded motion profile ends. The vertical line at 342 $\mathrm{ms}$ indicates the time when the servo has settled to the goal of $\pm 3 \mathrm{~nm}$ of the commanded position. When examining the data in figure 5 before the $154 \mathrm{~ms}$ mark, significant inertial response is observed. This is expected because, during this time the servo is commanded to rapidly accelerate and decelerate. Also, during the gross motion of the servo, the frictional response appears as a nearly constant drag on the system. Although it does not have the ideal qualities of a theoretical representation, (a constant frictional force while moving in one direction), it does resemble the expected action of Coulomb friction. The time between $154 \mathrm{~ms}$ and $342 \mathrm{~ms}$ is the settling phase of the step and settle process. An interesting observation from this phase is that the inertial response of the system appears to be very small while the frictional response is dominant. This leads to a key proposition: during servo settling, there will be a point where the system response will be almost exclusively frictional and the inertial response of the servo will be insignificant. For this servo study, the proposition seems to take effect as soon as the commanded motion profile ends. For other servos or other motion profiles, this may not be the case, for example motion trajectories with large instantaneous accelerations or systems with very low friction, but as the servo settles, the proposition will eventually hold.

Continuing the study of the servo settling, the position error data from the step motion is plotted versus the input force and force of friction in figure 6. The partial loop structure, seen in figure 6, also suggests that the system response has characteristics that could be captured by a parameterization of the Dahl model. In fact, other work by the authors show that this behavior can be used to identify friction in servo mechanisms [1, 2]. In light of the previous observation that friction can dominate the servo response, for now, let it be assumed that the sufficient governing equation for the controlled plant during settling is

$$
F_{r}=u(t)
$$



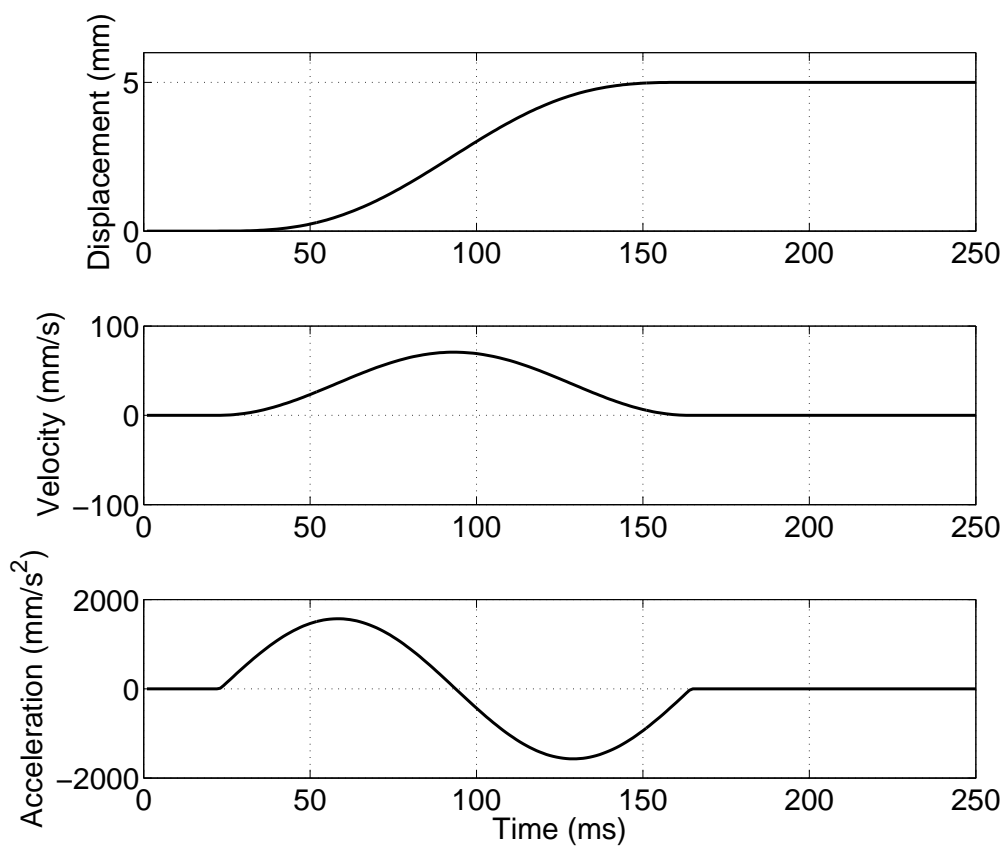

Fig. 4. This plot shows the commanded displacement, commanded velocity, and the commanded acceleration for a typical 5 $\mathrm{mm}$ move-and-settle operation for the servo.

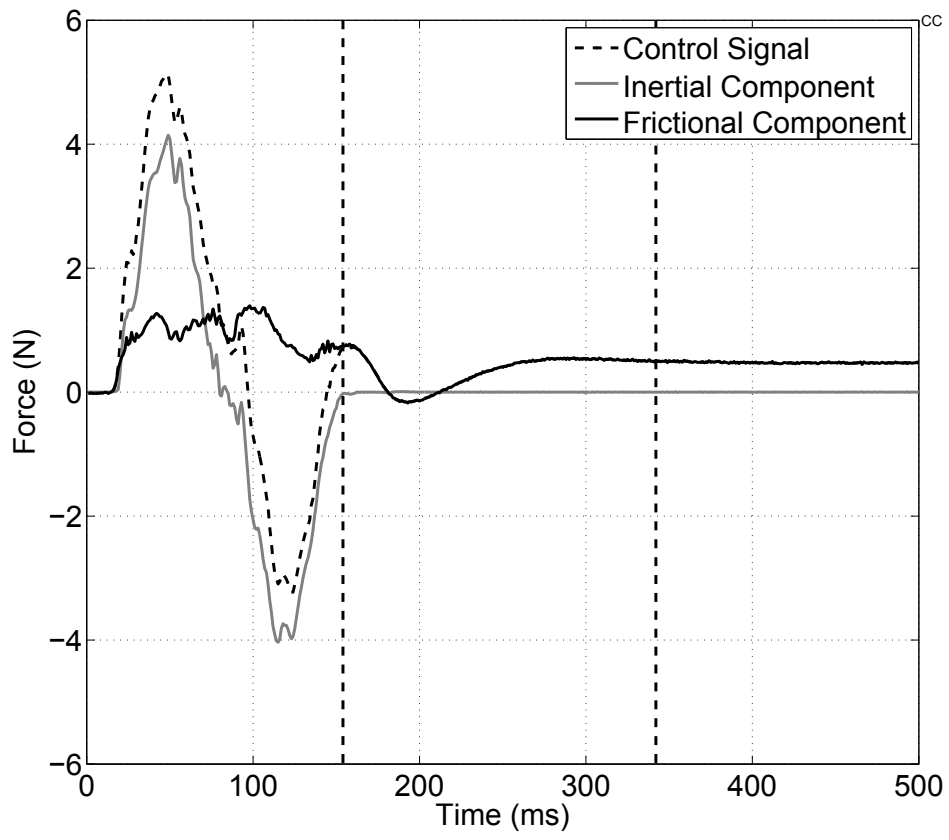

Fig. 5. Input signal for a typical $5 \mathrm{~mm}$ step motion being decomposed into the two primary dynamics (inertial response and frictional response) of the servo. 


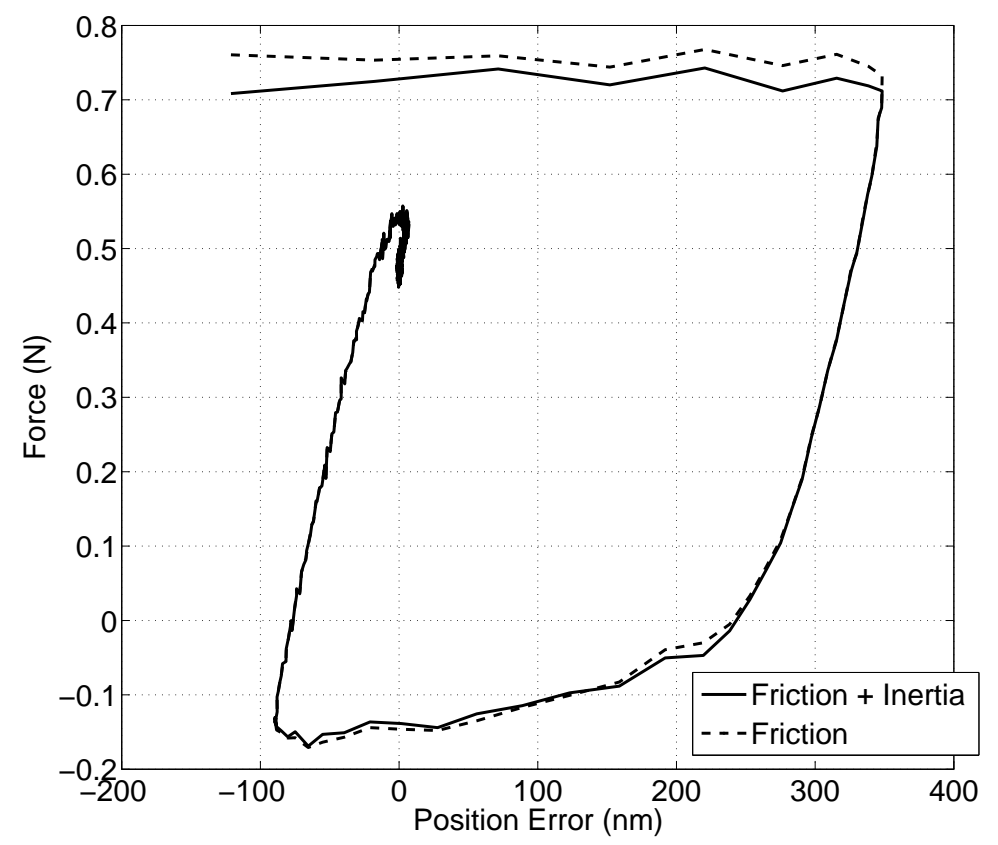

Fig. 6. This figure presents force versus displacement data from the settling portion of step motion shown in figure 5 .

where $F_{r}$ is a parameterization of the Dahl model and $u(t)$ is the control signal. Although there are cases for which this approximation will not be valid, it will be shown later that it is realistic and useful.

\section{Formulating the NiAS A Control LaW}

In the following section, the proposed servo settling algorithm is detailed, followed by a description of the resulting error dynamics equations and consideration of uncertainty in the friction process.

\section{A. Linear PI Control Formulation}

The linear PID control law will be developed first. Its performance will be contrasted to the proposed algorithm. As stated earlier, the approximate plant during final servo settling is simply the frictional process (equation 4), which will be used for the initial control development. In the regime that we are most interested in, the dynamics of the forces that relate to friction dwarf those related to inertia, and thus the inertia is neglected. Once the massless approximation is 
used to derive the controller, an analysis that includes the inertial response of the servo will follow.

Let the position error signal be defined as

$$
e(t)=r(t)-x(t)
$$

where $r(t)$ is the position reference. Since the reference signal is constant during the settling process,

$$
\dot{e}(t)=-\dot{x}(t)
$$

Integral control of the plant can be expressed as

$$
F_{r}(x)=u(t)=\int_{0}^{t} k_{I} e(t) d t,
$$

where $k_{I}$ is the integral gain. Most often the constant $k_{I}$ would appear before the integral but, in the current case, is shown inside the integral for later purposes. To achieve a more convenient form equation 7 can be differentiated with respect to time yielding,

$$
\frac{d F_{r}}{d t}=\frac{d F_{r}}{d x} \frac{d x}{d t}=k_{I} e
$$

This gives the error dynamics equation of

$$
\frac{d F_{r}}{d x} \dot{e}+k_{I} e=0
$$

or

$$
\dot{e}=-\frac{k_{I}}{\frac{d F_{r}}{d x}} e .
$$

Since $k_{I}>0$ and $\frac{d F_{r}}{d x}$ always has the same sign as the direction of motion the approximate system is stable but nonlinear. Extending the concept of integral control to PI control leads to the error dynamics equation of

$$
\left(\frac{d F_{r}}{d x}+k_{P}\right) \dot{e}+k_{I} e=0
$$

or

$$
\dot{e}=-\frac{k_{I}}{\frac{d F_{r}}{d x}+k_{P}} e,
$$


where $k_{P}$ is a proportional gain. An interesting observation from equation 12 is that if $k_{P}$ were to become much larger than $\frac{d F_{r}}{d x}$, the contribution of the nonlinearity to the system response will be minimized. This case is an example of linearizing a system with high gain feedback. In practice, the gain $k_{P}$ is limited due to uncertainties in the plant and required levels of robustness of the controller.

\section{B. Nonlinear Control Algorithm}

This section presents a practical method of producing a linearized response from the nonlinear closed loop plant. Considering the case of PI control, suppose that $k_{I}$ is not a constant, instead, it is defined by the function

$$
k_{I}(x)=\frac{1}{\tau_{d}}\left(\frac{d F_{r}}{d x}+k_{P}\right),
$$

where $\tau_{d}$ is a design time constant, which controls the rate of decay for the error (theoretically a first order error dynamic). This method will be referred to as the Nonlinear Integral Action Settling Algorithm (NIASA). This nonlinear integral gain is instantaneously set to a large value with each direction change, but asymptotically levels off after a short distance, similar to the Dahl model in Figure 3. Although the NIASA is on all the time, it largely only affects settling performance, which is characterized by multiple direction changes. Applying this result to equation 12 leads to linear first order error dynamics of

$$
\dot{e}=-\frac{1}{\tau_{d}} e .
$$

The result seen in equation 14 is favorable from a theoretical perspective. However, there are two important issues that need to be addressed. First is robustness; this result implies that the model of the friction process exactly matches the true friction process. A more realistic interpretation of the error dynamics in equation 14 would be

$$
\dot{e}=-\frac{1}{\tau_{d}} \frac{\frac{d \hat{F}_{r}}{d x}+k_{P}}{\frac{d F_{r}}{d x}+k_{P}} e .
$$

where $\frac{d \hat{F}_{r}}{d x}$ denotes the nominal differential model of the friction process. Second is the small inertia assumption; the plant under consideration is still an approximation of the true plant and the inertial response of the servo is not considered. It appears that the massless approximation is valid under low acceleration conditions but, it is clear that it would not be valid under conditions 
with greater accelerations (perhaps $\ddot{x} \geq F_{r} / 10 m$ in equation 3). A more accurate representation of the servo error dynamics is

$$
m \dddot{e}+k_{D} \ddot{e}+\left(\frac{d F_{r}}{d x}+k_{P}\right) \dot{e}+\frac{1}{\tau_{d}}\left(\frac{d \hat{F}_{r}}{d x}+k_{P}\right) e=0,
$$

where $m$ is the mass or moment of inertia of the servo and $k_{D}$ is a derivative gain. The values for $k_{D}$ and $k_{P}$ come from a conventional linear PID tuning of the servo.

Thus, considering equation 13 and using the approximation of equation 2 that will be covered later, the proposed nonlinear PID control law is stated as

$$
u(t)=\frac{1}{\tau_{d}} \int_{0}^{t}\left(\frac{d \hat{F}_{r}}{d x}+k_{P}\right) e(t) d t+k_{P} e(t)+k_{D} \dot{e}(t),
$$

When considering control of the massless approximate plant, the control law is the same as equation 17, except $k_{D}=0$.

\section{Development of Error Dynamics Equations}

To study the behavior of the proposed NIASA method a realistic test scenario is to be constructed. This section aims to first, describe how the equations are to be set up to analyze this problem. Then, the nature of the test and how it relates to the actual servo settling problem are discussed.

1) Expressing the Friction Model in terms of Position Error: The Dahl model contains the sign function which complicates analysis. To deal with this, the Dahl model, with $i=1$, is considered as two functions, one for motion in the positive direction

$$
{\frac{d F_{r}}{d x}}^{(+)}=\sigma\left(1-\frac{F_{r}}{F_{C}}\right),
$$

and the other for motion in the negative direction

$$
{\frac{d F_{r}}{d x}}^{(-)}=\sigma\left(1+\frac{F_{r}}{F_{C}}\right) .
$$

Equations 18 and 19 can be separated and integrated to yield

$$
F_{r}(x)^{(+)}=F_{C}-\left(F_{C}-F_{0}\right) \exp \left(-\frac{\sigma}{F_{C}}\left(x-x_{0}\right)\right),
$$


and

$$
F_{r}(x)^{(-)}=-F_{C}+\left(F_{C}+F_{0}\right) \exp \left(\frac{\sigma}{F_{C}}\left(x-x_{0}\right)\right),
$$

where $F_{0}$ is the initial condition of the force of friction and $x_{0}$ is the initial condition on displacement. When simulating the frictional process, one switches between these two equations based on the direction of motion. The initial conditions seen in equations 20 and 21 come from the final states of the previous equation when a velocity reversal occurs. If the system has been traveling in one direction for a sufficient amount of time, $F_{0}$ would be the corresponding level of Coulomb friction. If you did not exit the pre-rolling regime, (very small step), $F_{0}$ would be whatever value is predicted by the friction model. It is interesting to note that the NIASA method does not require extremely accurate modeling of the friction state to achieve favorable results, as will be discussed in more detail later in the paper. Equations 20 and 21 can be differentiated with respect to $x$ to yield

$$
{\frac{d F_{r}}{d x}}^{(+)}=\sigma \frac{F_{C}-F_{0}}{F_{C}} \exp \left(-\frac{\sigma}{F_{C}}\left(x-x_{0}\right)\right),
$$

and

$$
{\frac{d F_{r}}{d x}}^{(-)}=\sigma \frac{F_{C}+F_{0}}{F_{C}} \exp \left(\frac{\sigma}{F_{C}}\left(x-x_{0}\right)\right) .
$$

Since $e=r-x$, the initial conditions can be expressed as

$$
e_{0}=r_{0}-x_{0}
$$

Since it is desired to study servo behavior during settling, where the position reference signal is no longer changing, let $r=r_{0}=$ constant. Equations 20 through 23 can now be reexpressed in terms of position error as

$$
\begin{gathered}
F(e)^{(+)}=F_{C}-\left(F_{C}-F_{0}\right) \exp \left(-\frac{\sigma}{F_{C}}\left(-e+e_{0}\right)\right), \\
F(e)^{(-)}=-F_{C}+\left(F_{C}+F_{0}\right) \exp \left(\frac{\sigma}{F_{C}}\left(-e+e_{0}\right)\right), \\
{\frac{d F_{r}}{d x}}^{(+)}=\sigma \frac{F_{C}-F_{0}}{F_{C}} \exp \left(-\frac{\sigma}{F_{C}}\left(-e+e_{0}\right)\right),
\end{gathered}
$$


and

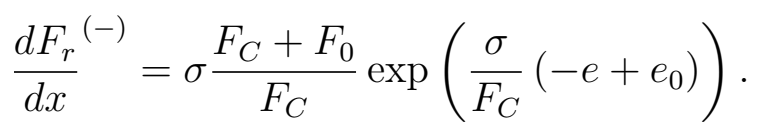

Thus, the friction model can be expressed in terms of position error alone.

2) Consideration of Uncertainty: One of the greatest difficulties in friction modeling and compensation is that the friction model, no matter how complex, is never able to capture all of the frictional behavior at all times. To study the effects of modeling error on the NIASA compensator in the most relevant, yet easily presentable way, uncertainty of the initial contact stiffness parameter, $\sigma$, will be considered since it was found to govern the stability of the algorithm $[1,2]$. In experimental studies (while keeping operating conditions reasonably constant), variations of nearly a factor of four in the initial contact stiffness parameter have been measured for a single precision servo [2]. The variation in $\sigma$ was observed while running the experiments for a number of trials, holding most environmental variables reasonably constant. It is prohibitive to include all the factors that can cause variations in friction. Even under relatively constant environmental conditions, frictional parameters can be identified only within some range and thus the control must be designed to operate effectively across this range of parametric variation.

Let a multiplicative uncertainty of the initial contact stiffness be considered as

$$
\sigma^{*}=\sigma(1+\delta)
$$

where $\delta$ is an uncertainty parameter. The reader is referred to other references regarding experimental methods to identify the friction parameters $[1,2]$. In experimental observations using the Aerotech ALS-130-H linear stage, the initial contact stiffness of the servo under test showed a mean value near $8 \mathrm{~N} / \mu \mathrm{m}$ with a minimum value of $4 \mathrm{~N} / \mu \mathrm{m}$ and a maximum value of $16 \mathrm{~N} / \mu \mathrm{m}$. If the nominal value of the initial contact stiffness is set to the observed mean value of $8 \mathrm{~N} / \mu \mathrm{m}$, this leads to the parametric uncertainty being bounded as

$$
-0.5 \leq \delta \leq 1
$$

Equations 25 through 28 are modified to become

$$
F(e)^{(+)}=F_{C}-\left(F_{C}-F_{0}\right) \exp \left(-\frac{\sigma(1+\delta)}{F_{C}}\left(-e+e_{0}\right)\right),
$$




$$
\begin{gathered}
F(e)^{(-)}=-F_{C}+\left(F_{C}+F_{0}\right) \exp \left(\frac{\sigma(1+\delta)}{F_{C}}\left(-e+e_{0}\right)\right), \\
{\frac{d F_{r}}{d x}}^{(+)}=\sigma(1+\delta) \frac{F_{C}-F_{0}}{F_{C}} \exp \left(-\frac{\sigma(1+\delta)}{F_{C}}\left(-e+e_{0}\right)\right),
\end{gathered}
$$

and

$$
{\frac{d F_{r}}{d x}}^{(-)}=\sigma(1+\delta) \frac{F_{C}+F_{0}}{F_{C}} \exp \left(\frac{\sigma(1+\delta)}{F_{C}}\left(-e+e_{0}\right)\right) .
$$

3) Equations of Error Dynamics Considering Uncertainty: With the results of the previous subsections, frictional dynamics of servo settling can now be expressed in terms of position error. Also, a means to consider friction model error has been developed. These results can now be used to study the error dynamics described by the massless approximation shown in equation 15 and the case considering mass shown in equation 16. Equations 15 and 16 contain the term $\frac{d \hat{F}_{r}}{d x}$ which refers to the nominal model of the friction process. In the context of this discussion, this corresponds to the case where $\delta=0$. Thus, the error dynamics for the massless approximation can be expressed as

$$
\dot{e}=-\frac{1}{\tau_{d}} \frac{\sigma \frac{F_{C}-\hat{F}_{0}}{F_{C}} \exp \left(-\frac{\sigma}{F_{C}}\left(-e+e_{0}\right)\right)+k_{P}}{\sigma(1+\delta) \frac{F_{C}-F_{0}}{F_{C}} \exp \left(-\frac{\sigma(1+\delta)}{F_{C}}\left(-e+e_{0}\right)\right)+k_{P}} e
$$

for motion in the positive direction and

$$
\dot{e}=-\frac{1}{\tau_{d}} \frac{\sigma \frac{F_{C}+\hat{F}_{0}}{F_{C}} \exp \left(\frac{\sigma}{F_{C}}\left(-e+e_{0}\right)\right)+k_{P}}{\sigma(1+\delta) \frac{F_{C}+F_{0}}{F_{C}} \exp \left(\frac{\sigma(1+\delta)}{F_{C}}\left(-e+e_{0}\right)\right)+k_{P}} e
$$

for motion in the negative direction. The initial force condition for the model of the friction process, $\hat{F}_{0}$, has the hat because the model of the friction process may not have the same initial force condition as the true friction process. It can be shown through a Lyapunov analysis that the robust stability condition for the massless case is $-1<\delta<\infty$ which is in agreement with equation (30). Similarly, the error dynamics equation when considering mass and motion is in 
the positive direction is

$$
\begin{array}{r}
m \dddot{e}+k_{D} \ddot{e}+\left(\sigma(1+\delta) \frac{F_{C}-F_{0}}{F_{C}} \exp \left(-\frac{\sigma(1+\delta)}{F_{C}}\left(-e+e_{0}\right)\right)+k_{P}\right) \dot{e}+ \\
+\frac{1}{\tau_{d}}\left(\sigma \frac{F_{C}-\hat{F}_{0}}{F_{C}} \exp \left(-\frac{\sigma}{F_{C}}\left(-e+e_{0}\right)\right)+k_{P}\right) e=0,
\end{array}
$$

and for motion in the negative direction is

$$
\begin{aligned}
m \dddot{e}+k_{D} \ddot{e}+(\sigma(1 & \left.+\delta) \frac{F_{C}+F_{0}}{F_{C}} \exp \left(\frac{\sigma(1+\delta)}{F_{C}}\left(-e+e_{0}\right)\right)+k_{P}\right) \dot{e}+ \\
& +\frac{1}{\tau_{d}}\left(\sigma \frac{F_{C}+\hat{F}_{0}}{F_{C}} \exp \left(\frac{\sigma}{F_{C}}\left(-e+e_{0}\right)\right)+k_{P}\right) e=0 .
\end{aligned}
$$

With a pair of error dynamics equations developed for the massless approximation and an approximation including the mass of the servo, it is now necessary to discuss switching between the equations (at velocity reversal) in each pair and applying the proper initial conditions. To find the new force initial condition, either equation 31 or equation 32 (depending on the direction of motion before the velocity reversal) is solved using the original force initial condition, $F_{0}$, the original position error initial condition, $e_{0}$, and the value of $e$ is set to the position error at the most recent velocity reversal. The resulting value of $F(e)$ becomes the new force initial condition. As previously mentioned, the modeled value of $\hat{F}_{0}$ may be different from $F_{0}$ in the true friction process. Thus, these parameters must be solved separately and using the proper values for $\delta$.

\section{Simulation}

In this section, we study the behavior of a servo system using the NIASA control law using numerical simulation. The aim of this test is to simulate servo behavior in the final stage of ultra-precision settling, since this is where the slowing of the settling process appears most dramatic. The simulations will start from rest at a distance of $62.5 \mathrm{~nm}$ from the target location. The parameters used in the simulation are chosen from the estimated physical parameters and factory PID gains from an actual precision linear stage. Table I gives the parameters used in the 
TABLE I

PARAMETERS USED IN THE SETTLING SIMULATIONS.

\begin{tabular}{c|c}
\hline \hline Parameter & Value \\
\hline$m$ & $1.5 \mathrm{~kg}$ \\
$\hat{\sigma}$ & $8 \mathrm{~N} / \mu \mathrm{m}$ \\
$F_{C}$ & $1 \mathrm{~N}$ \\
$k_{P}$ & $0.8 \mathrm{~N} / \mu \mathrm{m}$ \\
$k_{D}$ & $8.50 \times 10^{-4} \mathrm{Ns} / \mu \mathrm{m}$ \\
$\tau_{d}$ & $4 \mathrm{~ms}$ \\
$\sigma$ & {$[4 \mathrm{~N} / \mu \mathrm{m}, 16 \mathrm{~N} / \mu \mathrm{m}]$} \\
$\delta$ & {$[-0.5,1]$} \\
$e_{0}$ & $62.5 \mathrm{~nm}$ \\
$\dot{e}_{0}$ & 0 \\
$\ddot{e_{0}}$ & 0 \\
$\ddot{e_{0}}$ & 0 \\
$F_{0}$ & $0 \mathrm{~N}$ \\
$\hat{F}_{0}$ & $0 \mathrm{~N}$ \\
\hline
\end{tabular}

simulations.

\section{A. Simulation Results With and Without Mass}

Simulations of the massless system (eqs. 35 and 36) and the system including mass (eqs. 37 and 38) were conducted in MATLAB, using a 4th order Runge-Kutta method. Time constants of $\tau_{d}=4$ and $10 \mathrm{~ms}$ were simulated. The $\tau_{d}=10 \mathrm{~ms}$ case provided little difference between the case with and without the mass, so the more aggressive design time constant was chosen. Figure 7 shows the results of simulating the massless approximation for $\delta=\{-0.5,0,0.5,1\}$. Figure 8 shows the results of the simulation including the mass of the servo for the same values of $\delta$. The ideal response is also included in Figure 8 for comparison. Incidentally, the $\delta=0$ case in Figure 7 is identical to the ideal response. The most encouraging observation from figures 7 and 8 is that all of the simulations appear to be converging to zero steady state error in a similar amount of time in spite of the uncertainty of the initial contact stiffness parameter. When comparing figures 7 and 8 it is apparent that the massless approximation does provide a reasonable estimate of how the system will behave when mass is considered. This would suggest that the concept of the NIASA compensator developed in the massless approximation translate into a more realistic interpretation of servo motion which includes mass. The only significant 


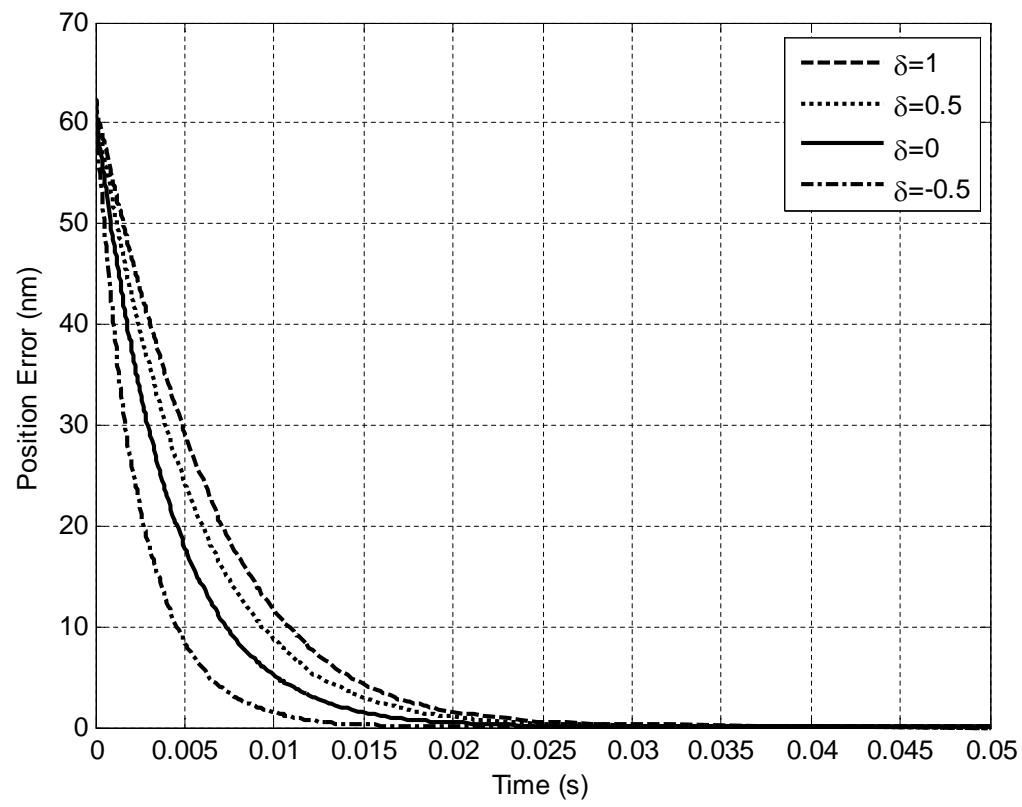

Fig. 7. Settling results of the massless closed loop simulations of NIASA compensator for $\delta=\{-0.5,0,0.5,1\}$ and $\tau_{d}=4$ ms.

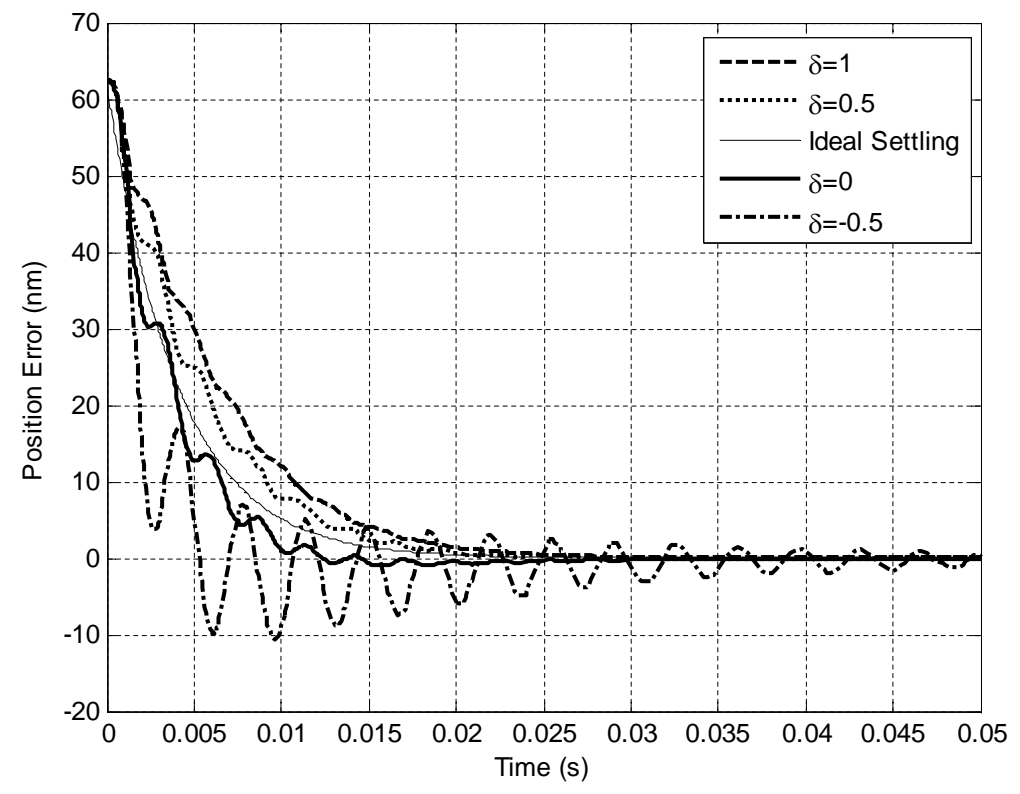

Fig. 8. Settling results of the closed loop simulations of the NIASA compensator including the mass of the servo for $\delta=$ $\{-0.5,0,0.5,1\}$ and $\tau_{d}=4 \mathrm{~ms}$. (To be compared with the massless simulations shown in figure 7. .) 
difference is seen in the case where $\delta=-0.5$, the case where the actual initial contact stiffness is $50 \%$ less than the modeled initial contact stiffness. In this case, the massless simulation predicts increased settling rate but, the simulation including mass shows significant ringing, which leads to a decrease in settling rate. This ringing is due to a combination of increased inertial response due to the aggressive design time constant, $\tau_{d}$, and the over estimation of the initial contact stiffness parameter. Since both of these parameters enter the error dynamics equations at nearly the same point, their respective effects can be difficult to separate. This effect is analogous to a reduction in damping seen in a second-order linear system, under PID control, when the integral gain may be set too high.

The key concepts to be taken from examining these simulations are not really related to the specific numerical values produced by the simulations. Rather, it has been shown that approximating a servo as only the frictional process is valid under certain circumstances. Control of this nonlinear approximate plant can be formulated to yield a nearly linear response (equation 14), even in the presence of parametric uncertainty. Finally, it is seen that utilizing the approximate plant and the NIASA compensator appears to provide a method of quickly driving the servo to zero steady state error even when the inertial dynamics are considered.

\section{EXPERIMENTAL RESULTS}

The simulation results presented in the previous section are promising. A brief experimental study of the proposed NIASA compensator was conducted on a ALS-130-H precision servo by Aerotech, Inc. Figure 9 shows a picture of the precision servo stage which has $100 \mathrm{~mm}$ of linear travel and an encoder resolution of $61 \mathrm{pm}$. Linear crossed roller bearings support the carriage, which has a moving mass of $1.8 \mathrm{~kg}$. The stage is driven by an NDrive ML Linear Controller/Drive by Aerotech. This gives a servo sampling rate of $8 \mathrm{kHz}$. Equations 17, 27, and 28 were used to implement the algorithm. Each trial consisted of 250 instances of $5 \mathrm{~mm}$ steps, using the motion profile described in figure 4. Since the algorithm was designed to take into account realistic conditions, the steps do not all start or stop in the same location. The stage was simply commanded to move up and down the range of travel of the device. The first trial was conducted with the factory tuned PID controller and the second was conducted using the same PID tuning combined with the NIASA compensator. The factory tuning was judged as acceptable by an experienced control system engineer and thus, provides a useful benchmark 


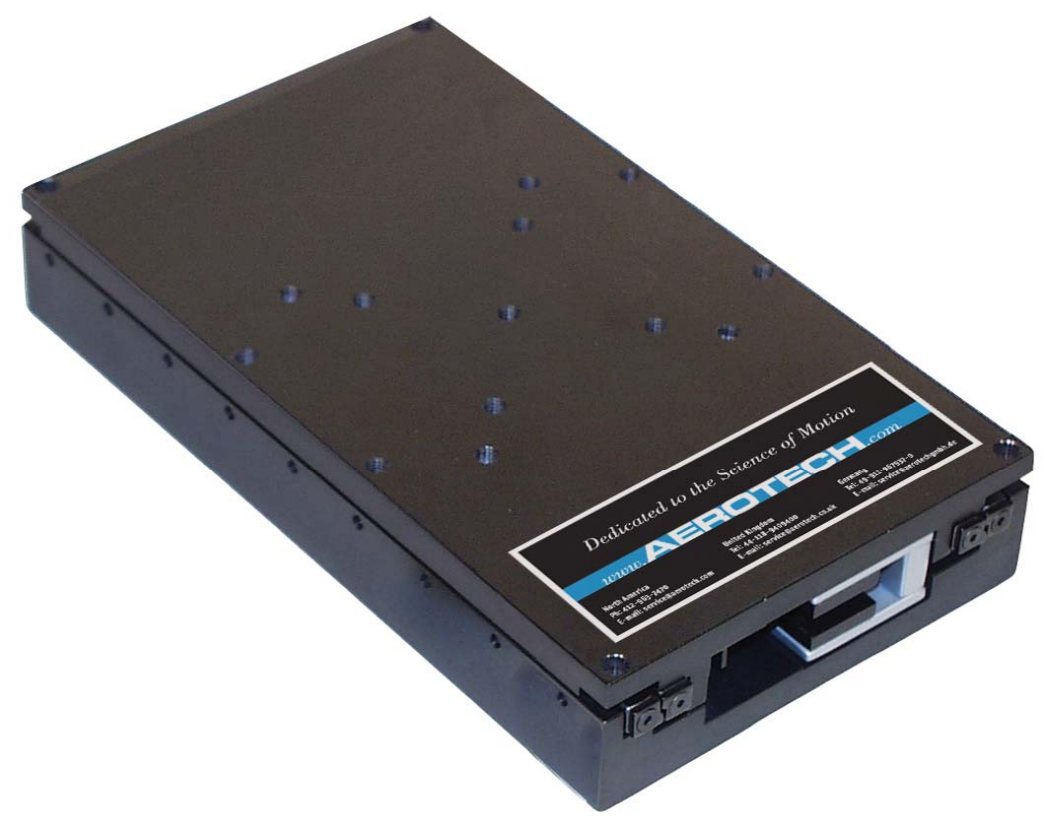

Fig. 9. Aerotech, Inc. ALS-130 Stage used for experimental demonstrations

for system performance. In interest of avoiding the undesirable oscillations seen in some of the simulations with the more aggressive parameterization of the NIASA compensator, the slightly less aggressive values of $\hat{\sigma}=4 \mathrm{~N} / \mu \mathrm{m}$ and $\tau_{d}=10 \mathrm{~ms}$ were used. The system was observed to have a mean value of Coulomb friction of $0.62 \mathrm{~N}$. Since $F_{C}$ doesn't have to be exact, a value of $F_{C}=0.6 \mathrm{~N}$ was used in the algorithm [1,2].

Figure 10 shows the mean settling times, to tolerances between $\pm 3 \mathrm{~nm}$ and $\pm 100 \mathrm{~nm}$, for the PID controller and the PID controller combined with the NIASA compensator. The dashed lines indicate one standard deviation from the mean value. Across all settling tolerances, the addition of the NIASA compensator is able to reduce the servo settling time by between $80.5 \%$ to $87.4 \%$. It is very encouraging that such significant improvement in servo settling performance can be accomplished with the addition of this algorithm to the PID controller however, examination of the position error signal is desirable to confirm the validity of the theory behind the NIASA compensator. Figure 11 shows typical series of position error versus time, during settling, for the PID, PID+NIASA, and a representation of ideal settling (equation 14), based on $\tau_{d}=10 \mathrm{~ms}$. As seen in figure 11, the PID controller tends to over shoot the target location several times and 


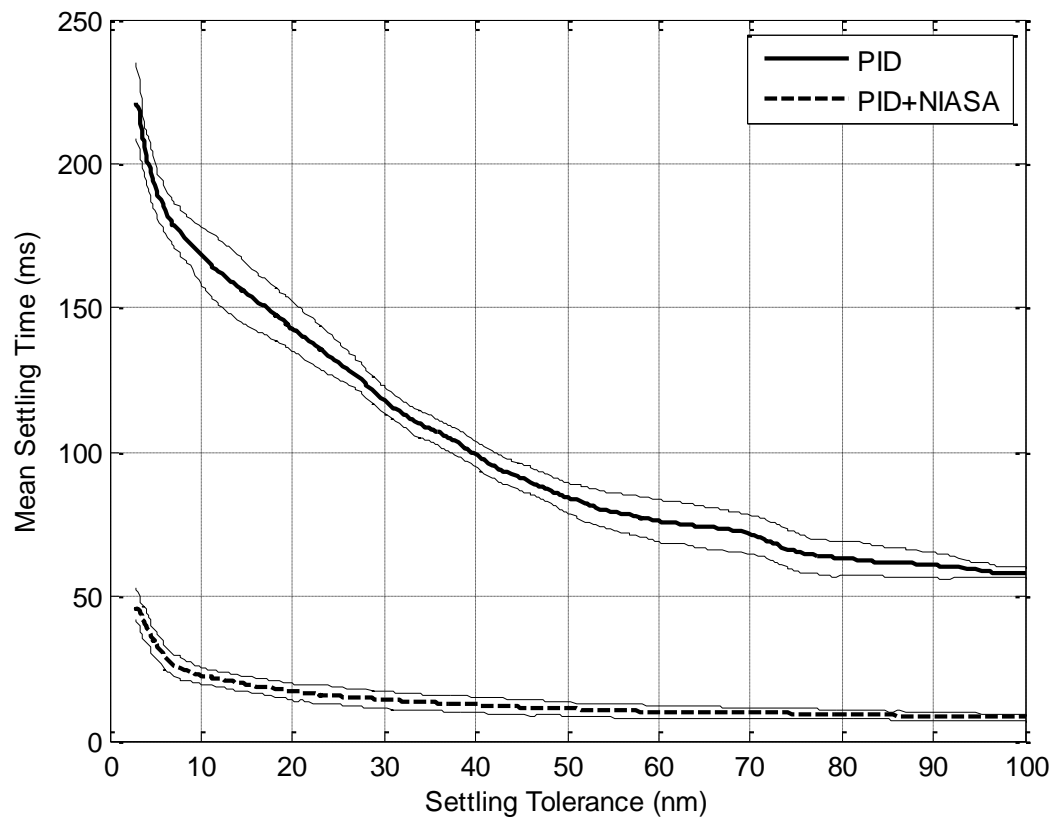

Fig. 10. Experimental settling performance for the PID controller and the same PID controller combined with the NIASA compensator.

spend a significant amount of time reversing direction to make another approach at the target location. This waveform appears very similar to that seen in figure 2 as the consequences of pre-rolling friction were being introduced. The time series for the PID+NIASA controller shows significantly faster settling, as compared to the PID controller by itself. It also does not have the issue with over shooting of the target location. Most interestingly, the PID controller combined with the NIASA compensator appears to converge to the target location with a trajectory quite similar to the ideal response that used the massless approximation.

\section{CONCLUSION}

When considering rapid point-to-point motion, where nanometer level precision is required, most conventional methods of friction compensation, (feedforward methods and friction observers), are not able to address the issues that pre-rolling friction causes in a way that makes a useful impact on servo settling performance. Using the observation that the final phase of servo settling tends to be dominated by pre-rolling friction, control for this approximate plant is proposed in the form of the Nonlinear Integral Action Settling Algorithm (NIASA). The design 


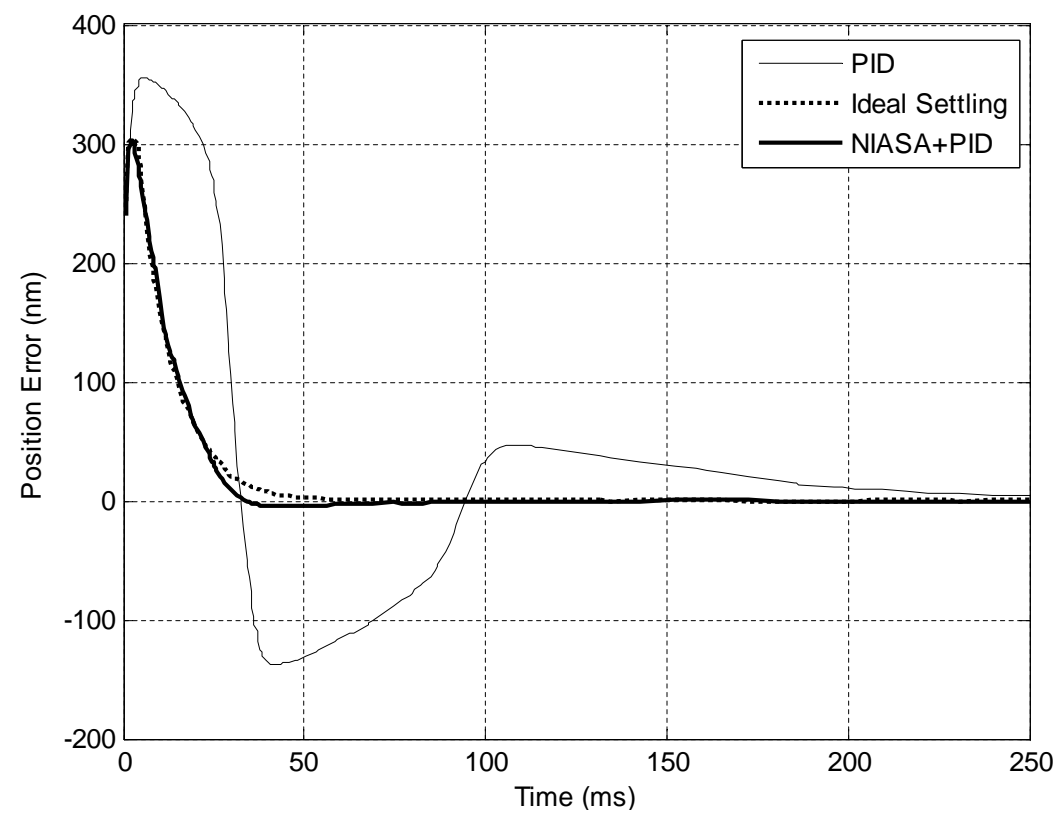

Fig. 11. This plot compares the typical settling behavior after a $5 \mathrm{~mm}$ step motion using only PID control and the same PID controller now combined with the NIASA compensator with $\tau_{d}=10 \mathrm{~ms}$.

of the NIASA compensator is done with consideration that friction can be an uncertain process (limiting the usefulness of feedforward and observer based control approaches). The simulations done in this study show the NIASA compensator being able to drive the servo to zero steady state error at similar rates even though there is significant difference between the modeled and true frictional parameters. Also, similar results can be achieved when the mass of the servo is considered or the servo is approximated as massless. Finally, an experimental study shows that the NIASA compensator is able to offer increased servo settling performance, by between $80.5 \%$ and $87.4 \%$, and that the concepts developed in the massless approximation of the servo appear to translate into actual practice.

\section{REFERENCES}

[1] B. Bucci, J.S. Vipperman, D. Cole, and S. Ludwick. Evaluating a servo settling algorithm. Precision Engineering, Revised Article Submitted.

[2] B. Bucci. A practical method for friction compensation in rapid point-to-point motion. 
$\mathrm{PhD}$ thesis, University of Pittsburgh, Department of Mechanical Engineering and Material Science, 2011.

[3] H. Olsson, K. Åstrom, C. Canudas de Wit, M. Gafvert, and P. Lischinsky. Friction models and friction compensation. European Journal of Control, 4:176-195, 1998.

[4] B. Armstrong-Helouvry, P. Dupont, and C. Canudas de Wit. A survey of models, analysis tools and compensation methods for the control of machines with friction. Automatica, 30:1083-1138, 1994.

[5] Friedhelm Altpeter. Friction Modeling, Identification, and Compensation. PhD thesis, Ecole Polytechnique Federale de Lausanne, 1999.

[6] C. Canudas de Wit and P. Lischinsky. Adaptive friction compensation with partially known dynamic friction model. International Journal of Adaptive Control and Signal Processing, 11:65-80, 1997.

[7] C. Canudas de Wit, H. Olsson, K. Åstrom, and P. Lischisnsky. A new model for control of systems with friction. IEEE Transactions on Automatic Control, 40(3):419-426, March 1995.

[8] H. Olsson and K. Åstrom. Observer-based friction compensation. In Proceedings of the 35th Conference on Decision and Control, 1996.

[9] H. Olsson. Control of Systems with Friction. PhD thesis, Lund Institute of Technology, 1996.

[10] F. Altpeter, P. Myszkorowski, M. Kocher, and R. Longchamp. Friction compensation: PID synthesis and state control. In European Control Conference, 1997.

[11] U. Schafer and G. Brandenburg. Model reference position control of an elastic two-mass system with backlash and Coulomb friction using different types of observers. Power Electronics and Motion Control, 3:797-801, 1990.

[12] G. Brandenburg and U. Schafer. Influence and partial compensation of simultaneously acting backlash and Coulomb friction in a speed and position controlled, elastic two-mass system. In Proceedings of the International Conference on Electrical Drives, 1988.

[13] I. Nilkhamhang and Akire Sano. Adaptive friction compensation using the GMS model with polynomial Stribeck function. Proceedings of International Conference on Control Applications, 2006.

[14] J. Martinez-Rosas and L. Alvarez-Icaza. Adaptive compensation of dynamic friction in an 
industrial robot. In 17th IEEE International Conference on Control Applications, 2008.

[15] P. Dahl. A solid friction model. Technical report, Space and Missile Systems Organization Air Force Systems Command, 1968. 